\title{
Research on the Present Situation, Problems and Countermeasures of the Architecture of Lingnan Flower-Bird-Fish Art Wholesale Market
}

\author{
Hu Kaiwen \\ Architecture Department, South China University of Technology, Guangzhou, China
}

Email address:

hukaiwen0826@163.com

To cite this article:

Hu Kaiwen. Research on the Present Situation, Problems and Countermeasures of the Architecture of Lingnan Flower-Bird-Fish Art Wholesale Market. American Journal of Civil Engineering. Vol. 5, No. 6, 2017, pp. 418-425. doi: 10.11648/j.ajce.20170506.25

Received: November 5, 2017; Accepted: November 23, 2017; Published: December 29, 2017

\begin{abstract}
Based on the historical background of the flower base of Fangcun in Guangzhou, this paper studies the development of its regional history, industry support and policy support. Taking Guangzhou Yuehe Flower-Bird-Fish Art World as an example, through field investigation, specific studies of the present situation and practical problems of market architecture are probed into. According to the shortcomings found in the research, specific countermeasures for the design of exterior and interior space of the building as well as the suggestions for humanity, information, legalization, brand innovation of the wholesale market are put forward.
\end{abstract}

Keywords: Fangcun Flower and Bird Wholesale Market, Architecture Status, Countermeasures

\section{Introduction}

The new type of wholesale business is exerting a great vitality in many western developed countries and even a more robust momentum in China even if its traditional mode is suffering a downtown [1]. Of all the wholesale businesses in China, the Flower-Bird market holds a unique position for it goes along well with the Chinese Four Arts-Music, Chess, Calligraphy and Painting, which have enriched the folk cultures. However, many wholesale markets have to be relocated due to aging facilities [2]. Beijing Guanyuan Flower-Bird market will be shut down to be relocated; Yongan Flower-Bird market has to be rebuilt by the government for being an eyesore to the city with so many small unregulated stalls [3]; After three years of sprucing up in Kunming, the Jewelry Flower-Bird market in its old street is ready for the soft opening [4]. All of the aforementioned examples well explain the existing problems in the flower and bird market. Meanwhile, it can serve as a good resort for leisure and also a prompter for surrounding business if the flower and bird market has a better environment [5]. As modern city should be a reflection of people's lives, flower \& bird market as a part of folk culture certainly can grow along with the urban development, and become an eye-catcher in the city [6].

In recent years, few theoretical studies in China have been directed at the architectural design of flower-bird markets, a special branch of the wholesale market. However, there are quite a few studies on the wholesale market. Some of them are studied from the perspective of architectural design. For instance, Xie Puqi and Zhao Yang takes Guangzhou Yide Road Professional Wholesale Market as an example, and does a research on the transformation and development of the traditional business streets with arcade buildings, which provides references for the transformation and development of other wholesale markets located in the historic downtown [7]. Ma Hongzhao makes an analysis of the main points of design for the large-scale commodity wholesale market [8]. Meng Zinan and Zhang Yupeng take the case of North China Wenzhou Mall in Gongzhuling City as an example to study the development status and countermeasures of the commercial wholesale markets [9]. Others are discussed from the perspective of building fire prevention. For example, Cui Yijun and Lu Feiyu make an analysis on the fire characteristics and fire prevention strategies in the commodity wholesale market [10]. Lin Yu proposes a design to optimize the evacuation of large wholesale markets [11]. Zhao Yifan and Li Xiaomin focus on fire safety of the small 
commodities wholesale markets, and puts forward some strategies, including constructing a leading system, enhancing the autonomous system, improving the regulatory system, and strengthening the fire safety publicity and training system [12].

\section{Historical Evolution}

Guangzhou, the capital of Guangdong province, is the famous "Flower City" in China, and the political, economic and cultural center of Lingnan. Fangcun, which is in Liwan District of Guangzhou, is the research center and production base of Lingnan' s flower and bonsai. It is known as the "First Flower Town in Lingnan" and "Flower City of the Flower City".

\subsection{Regional History}

\subsubsection{Origin of the Flower Market}

Why did Fangcun become a flower base in Guangzhou? According to the analysis of historical data, there are three main reasons for the amalgamation of flowers. The first reason is the need for flowers to worship Buddha. The second reason is the Datong port is an important port for generations, where the vessels from home and abroad often put in, bringing in exotic flowers and rare herbs. In addition, many flowers in Fangcun are brought by officials of Guangzhou. There are three ways to buy and enjoy flowers in, Guangzhou, such as Flower Exhibition, Flower Fair and Flower Market (visits). The Huaxu (Flower Fair) in Fangcun is the largest flower and tree sales and distribution center in Guangzhou area. The Huaxu began in Ming Dynasty and flourished in Qing Dynasty [13]. In Fangcun, there is a place called Huadi (花棣), which is famous for its abundant flowers and recorded in history. Because the former name of this place is not colloquial enough, people begin to call it Huadi（花地）, “The Land of Flowers" after times. With the development of commodity economy in the Ming and Qing Dynasties and population increase, cities and towns in the Pearl River Delta have become increasingly prosperous. The commodity economy further stimulated the development of the flowers and trees industry. The residents of Huadi, located on the south bank of the Pearl River in Guangzhou, have become the Jasminum grandiflorum growers [14]. Because of the geography, climate, economy and culture and so on, the flower market of Fangcun in Guangzhou is well-known far and wild.

\subsubsection{Garden Promotion}

Huadi is known as the "Hometown of Flowers", the "Hometown of Bonsai" and the "Hometown of Garden" in Lingnan in history. By the 20s of this century, bonsai artists in Fangcun resolutely broke through the shackles of traditional mode, and made radical changes in the style, conception and production skills of bonsai. In the late thirties, as a school of Chinese bonsai art, the Lingnan's bonsai has been a new force and has become the focus of the world. Fangcun became one of the birthplaces of Lingnan's bonsai art [8]. In Ming Dynasty Fangcun has witnessed the situation of dozens of garden pavilions, and numerous professionals in planting flowers and trees for the industry. In Qing Dynasty, gardens of flowers had reached its peak, of which the amount was more than 30. Among them, the famous Eight Gardens, with various features of their own, mainly sold flowers, trees and bonsai, meanwhile, for visits. Driven by the development of gardening, Fangcun is an option for more tourists.

\subsubsection{Traffic Advantages}

The location of Fangcun is superior. It is located in Guangzhou, the birthplace of the Maritime Silk Road, and has an important port, that is, Fangcun is near Datong Port, which was an important port in the west side of the White Goose Lake.

\subsection{Industrial Support}

In the 1980s, Fangcun, as an important flower production base, boasted of more than 960 kinds of flowers and trees planted. They were exported to Hong Kong, Macao, Europe, the United States and so on, which had become the main economic benefits of local farmers. In 1985, during the third construction of Fangcun District, the CPC Guangzhou Municipal Committee and the Municipal Government determined to build Fangcun District into a Flower City of the Flower City, and flower production in Fangcun District rose steeply. By the early 1990s, the flower planting area had reached more than 400 hectares, and flower seedlings were sold throughout the country.

\subsection{Policy Support}

In 2001, the government selected the Huadi Bay to build Flower-Bird-Fish-Worm Market, and provided the following policy support: the first one is Logistics. Guangzhou municipal government would vigorously support the development of large-scale professional wholesale market, promote the development of Liwan District's logistics, and increasingly improve the regional environmental facilities. Second is about attraction. Yuehe Flower-Bird-Fish Art World was designated as One Day Tour of Guangzhou by the Guangzhou Tourism Bureau at the end of 2003, which is one of the five major tourist attractions in Liwan. The third one is about the type of operation. With the joining of Golden Sea Horse and Bohuang Furniture Expo Center and other large stores, Huadi Bay in Fangcun has become the largest furnishings sales district in Guangzhou.

\section{Current Situation Analysis}

Yuehe Flower-Bird-Fish Art World (commonly known as the Fangcun Flower and Bird Wholesale Market) in Guangzhou occupies the favorable position at Pearl River Delta intersection combined with the historic landmarks such as Xiguan District, Shamian Island, Beijing Road Walking Street which are located on both sides of old Guangzhou Central Axis, and are at the throat of Guangzhou-Foshan metropolis circle, without traffic restrictions for nonlocal cars 
and adjacent to Fangcun passenger bus station. Fangcun Flower and Bird Wholesale Market is in Huadi Bay, Fangcun, Liwan district of Guangzhou city, located at Huadi Bay subway station's C and D exits of Guangzhou Metro Line 1 (Figure 1). The area embraces mainly Flower-Bird-Fish Art Wholesale Market, Pet Wholesale Market, Furniture Wholesale Market, Root Carving Wholesale Market. Through field research, the current situation and existing problems of Fangcun Flower and Bird Wholesale Market will be analyzed from the following aspects.
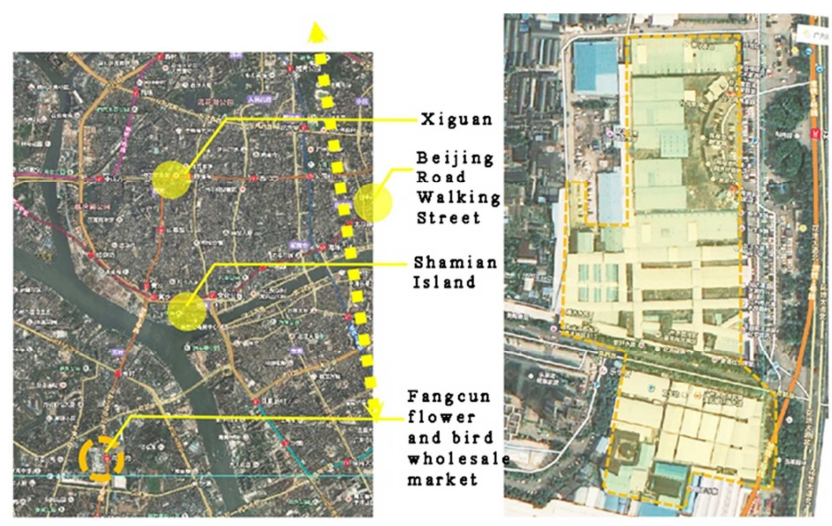

Figure 1. Analysis of Fangcun Flower and Bird Wholesale Market Location.

\subsection{Road and Streamline}

The surrounding roads: Main roads to Fangcun Flower and Bird Wholesale Market include Huadi Avenue on the east side of the market and the Xique road through the market. 3.3 kilometers long and 40 meters wide, Huadi Avenue, a north-south avenue, is the main road with two-way 8 lanes, a green belt in the middle, and is one of the eight entrances and exits of Guangzhou urban districts. Xique road in the east-west direction separates the market built in the first phase from the one in the second phase. It is found in the survey that the two roads are relatively smooth in normal times, but when approaching mealtimes Xique road is congested for a short while for many hawkers occupy the road and the sidewalk to sell their food.

The parking lots and auto streamline: The parking lots of Fangcun Flower and Bird Wholesale Market are located on the east of the plaza and inside the west area. The east one is larger and accommodates most of the nonlocal cars. The road to the west parking lot mainly serves for the large vehicles which are used to load and unload goods for the stores, but its narrowness is unfavorable for vehicles to drive in and out. There is a narrow traffic passage through the market, which connects Huadi Avenue and Xique road and also interlinks the inside parking lots. According to the research, the over-narrowness, too many windings and too much width changing of the path to the west parking lot do not satisfy two-way flows for the large freight vehicles. In addition, the small freight vehicles by the roadside impede the auto streamline. Besides, the eye-catching entrance to the parking lot is installed by an entrance device in customized airfoil shape (Figure 2) and equipped with card-taking window for collecting fee. There is more congestion in the east parking lot since it is built very early without making accurate prediction for the vehicle size and flow. Although the parking lot is one-way streamline, there is still no solution to the problems of the slow and chaotic parking lot.
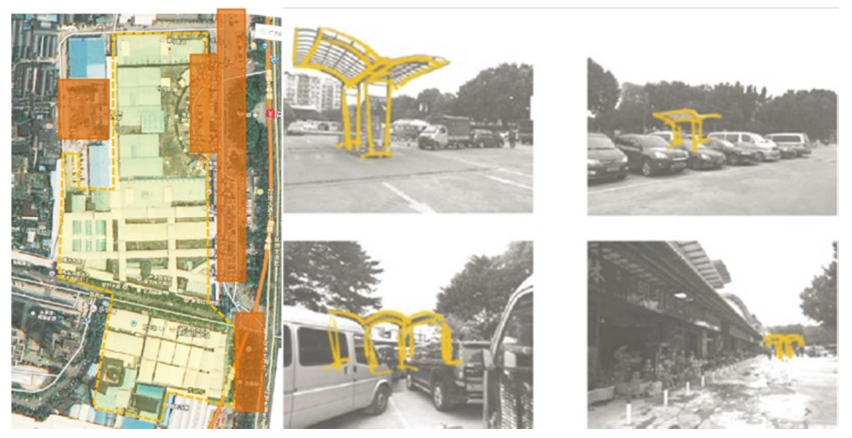

Figure 2. Parking layout and entrance shape.

Pedestrian streamline: The main stream of people in the Fangcun Flower and Bird Wholesale Market are merchants and customers. It's very important for merchants whether the streamline from the freight car parking lot to warehouses and to stores is smooth or not since it has impact on the time and labor cost of the sales. And it is also crucial for customers to visit the Fangcun Flower and Bird Wholesale Market whether the streamline of their sightseeing and shopping in the market is reasonable or not. West parking lot of the market mainly serves merchants as the transportation of goods. Main warehouses are in the northwest corner of the market and are near the west parking lot. But small warehouse, limited storage capacity and the different distance between stores manifest the weak storage function for the stores in the southeast corner. In general, pedestrian streamline is not convenient for merchants and the size and quantity of warehouses can't meet the requirements of the market. Customers going to the market are almost from Huadi Bay metro entrance and bus station, but it has a lot of entrances with the main entrance not highlighted and too many secondary entrances. Even though all entrances are marked only by the signs without any special or unique buildings to help pedestrians remember their location, pedestrians will be easy to get lost. What's worse is that the entrances are generally marked with parking space and will be occupied by vehicles which would bring obstacles to the sight and walking of pedestrians. The latticed passageway in the market, except the signs, fail to tell where they are. Without the loop of shopping streamline, customers cannot visit all the stores, which results in the waste of resources for the market.

It can be seen from the vehicle and pedestrian streamline that the market does not consider the separation of pedestrians and vehicles. Therefore, the intersection of vehicle flow and pedestrian flow is very congested, which reduces the transportation efficiency of merchants and at the same time poses a great hidden danger to the pedestrians. Especially at the junction, the coexistence of people and vehicles makes the traffic congestion more serious (Figure 3). 
Furthermore, other factors such as the unsuitable location of parking lots, insufficient area, the coexistence of people and vehicles and the prominent freight transportation problems, also lead to the lack of the convenience of the market.

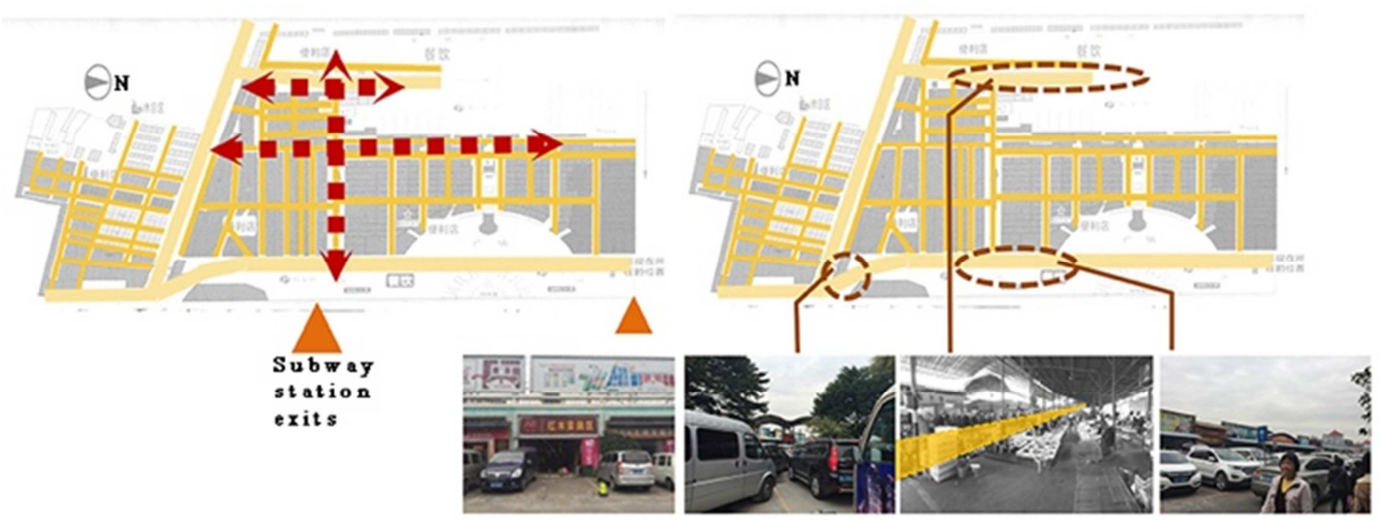

Figure 3. Streamline analysis, entrance modeling, congestion nodes.

\subsection{The Layout and Structure of Architecture}

Expansion process. The construction of Fangcun Flower and Bird Wholesale Market is not a one step process. It is divided into three stages. The first phase of the Aquarium Area in Flower-Bird-Fish-Worm Market opened on January 1, 2001 and gradually the Horticultural Area, the Bird Area and the Pet Area were constructed. In 2002, the second phase of the Flower-Bird-Fish-Art Market was developed and the top-grade Aquarium Area increased. Focusing on the word "art", the Household Handicrafts Area was gradually developed, and absorbed artificial flowers, ceramics, root carvings, calligraphy, paintings, collectibles, furniture and a variety of household industry businesses. With the combination of Flower-Bird-Fish-Worm Market (the first phase) with Flower-Bird-Fish-Art Market (the second phase), the market is called Yuehe Flower-Bird-Fish Art World, operating as an integrated market of home decoration. On the second half of 2006, with the impetus of wood and root carving industry drive, the Century Home Furnishings had transformed into mahogany classical furniture professional shopping malls, which makes the market known as "the best place for mahogany furniture". Therefore, a first-floor plan of the current market is formed (Figure 4).

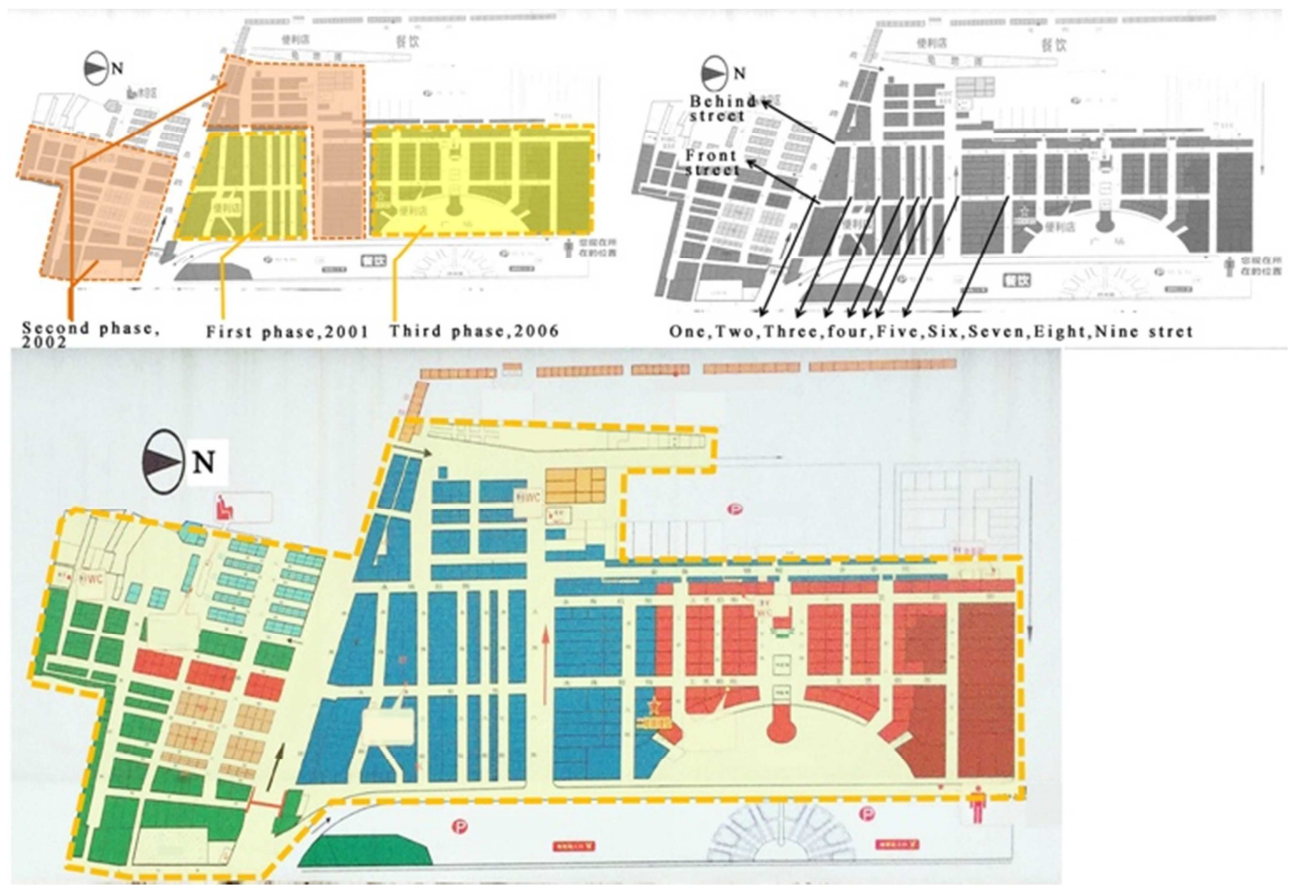

Figure 4. Expansion process, ground floor plan, road name.

Architectural layout. The expansion process shows that the plan layout of the functional space in the market is partitioned according to the expansion of the content. From north to south are Mahogany Furniture Area, Handicrafts
Area, Aquarium Area and Pet Area, Bird Area, Horticultural Area and Unusual stone area. After the research, it is found Aquarium Area covers the largest area, and is full of varieties, but the road changes in different directions. The complex 
interior space organization is dominated by east-west walkways, and in order to facilitate memory, the streets are named in terms of numbers from one to nine. The streets in south-north direction is named as the front and behind street.

Architectural structure. Due to the expansion in different years, there are two kinds of structures in the market construction. The Furniture Market is of load-bearing structure of building beams and columns; The Flowers-Bird-Fish-Art Market consists of bungalow storefront and the roof steel frame structure (Figure 5). The roofs are divided into single slope and arch. The Furniture Market has two floors, and the stairs and elevators are in the center area of the market, but the signs are not eye-catching, the stroll customers can miss them easily. And due to the limit store areas in the Flowers-Bird-Fish-Art Market, to show more, the roads are full of goods, which greatly reduce the width of the pedestrian. At the same time, the problems existing in the scene and the interior space of the market are flat and unattractive, and there is no consideration for later development and additional construction.
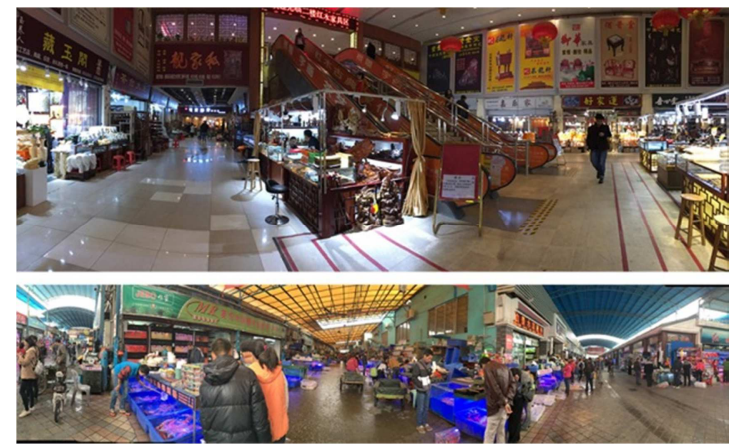

Figure 5. Beam-bearing structure, steel roof structure.

\subsection{Design Details}

Aisle width. Through field research, the aisle width in both Mahogany Furniture Area and Craft Area in Fangcun Flower and Bird Wholesale Market is approximately 4.8 meters $(8 \mathrm{x}$ 0.6 ), which is wide enough for the two-way freight carts to easily meet at the same time. There are usually less customers in Furniture Area, and a part of the stores are not open. Such a vast width makes the market seem more populousless. Some aquarium semi-indoor aisle width is approximately 6.2 meters. Without considering the separation of the goods flow from the people flow, and with businesses occupying the walkway area on both sides of the aisle, parts of the walkway are very congested and unsafe.

Staircases. Only Mahogany Furniture Area and Craft Area in the market have two layers. From the plane, the building only has two staircases. One is in the central district, and is equipped with two escalates; The other is a steeper staircase at the junction of Mahogany Furniture Area and Aquarium Area (Figure 6). Although the second floor of the building is mainly office management area, and some stores are not open, there are still not enough staircases. In order to meet fire safety requirements of the whole market business in the future, the location and quantity of staircases should be carefully considered.

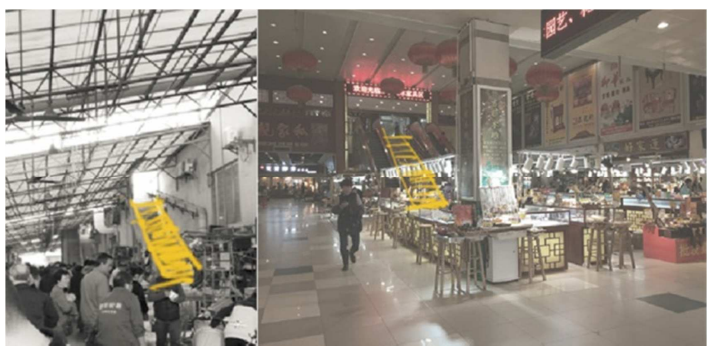

Figure 6. Stairs leading to the second floor.

Pipeline layout. There are problems in water supply and drainage in the market, because the goods in the Fangcun Flower and Bird Wholesale Market are living things, and the water demands of animals and plants is of a large quantity, especially in Aquarium Area which needs a lot of water supply and drainage for feeding and cleaning. But at the beginning of the architectural design there was no such consideration and it lead to the fact water supply and drainage facilities are somewhat primitive. Water pipe is crudely-connected with taps and there is only ditch drainage (Figure 7). Therefore, the grounds of the animals and plants district in Aquarium Area in the market cannot keep dry, which will bring inconvenience to the customers who come to have a look, and they cannot keep close contact with animals and plants while keeping their clothes clean. The sense of shopping experience needs to be strengthened. In addition, only Mahogany Furniture Area and Crafts Area in the market are equipped with air conditioning. In the original design, there is no such consideration for other areas, so fans are added in the sky in a semi-outer space in such places as Aquarium Area, with all kinds of pipelines, ventilation pipes, drains exposed, making the roof, wall, ground etc. of the buildings look unpleasant. At the same time, there are insufficient lighting in the semi-outer space in the market, and it is inconvenient for customers to choose goods when the sun is lacking in cloudy days and in the evening.
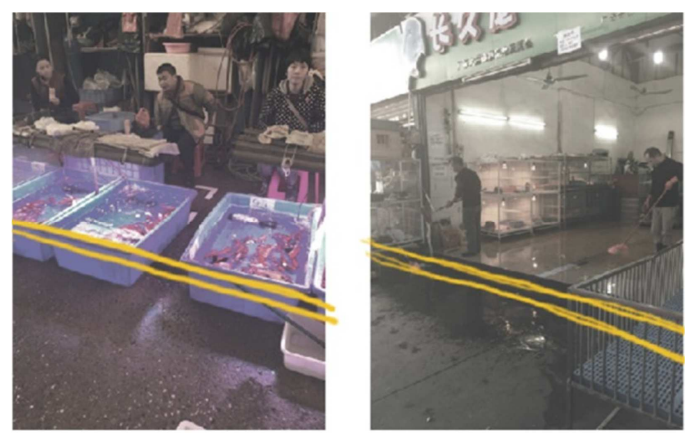

Figure 7. Drainage ditch.

\subsection{Supporting Facilities}

Groceries. There are a total of 4 groceries in the market (Figure 8), mainly set up for businessmen, and the goods there are daily groceries and snacks, which can only solve the urgent need of hunger, and cannot meet the needs of 
businessmen's daily meals. These stores are crowded, simply decorated and distributed in the market. And there are limited goods, and the conditions in the store are poor, which can only be called general stores or the snack bars, because there is a great gap with commonly recognized groceries.

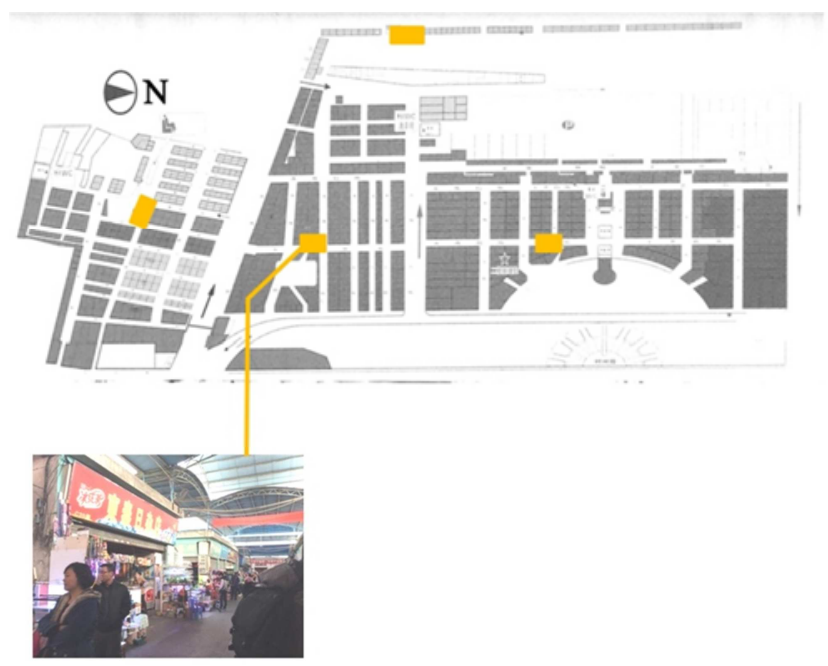

Figure 8. Groceries location.

Toilets. There are only four toilets in the whole Fangcun Flower and Bird Wholesale Market (Figure 9). Three are on the first floor, the other is on the second floor (which is being repaired and can't be used when we are doing the research). And the toilet is a building of single layer, which is divided into two rooms, one is for men and the other is for women. The toilets are scattered in the market, the area is narrow, each with 2-3 squats only, the light is dim, and the equipment is poor, which can only satisfy the basic need for merchants and customers to relieve themselves, and the comfort and cleanliness are not up to standard.

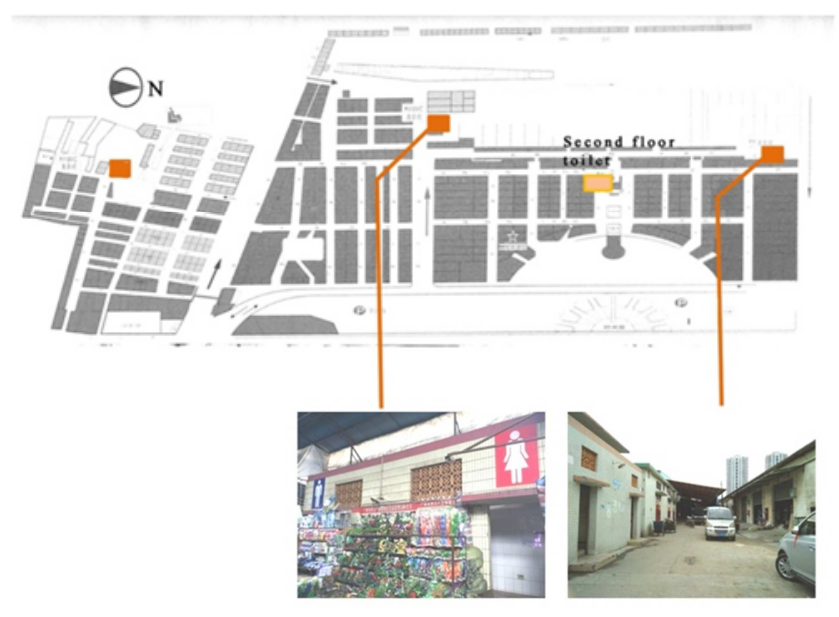

Figure 9. Toilets location.

Leisure service area. The only fast food restaurant on the east side of the market has no dining area, so food can only be taken outside. According to the interview, most of the businessmen would bring their own meals, order takeout, or buy food at a mobile stall. Customers are more difficult to have their meals, so they cannot stay in the market too long. In addition, there are still other problems such as not enough area for the customers to have a rest. The market only has a rest area in the southern side of the Bird Area (Figure 10). Most of the tourists would be tired before they arrive at the rest area. What's more, the equipment in the rest area is poor. At the same time, a very important part of the main customer source of the market is the elderly. Considering this factor, there is a serious lack of seats in the market, so suitable designs are urgently needed for the aging. While the garbage trucks in the market are collecting garbage from all businesses and keeping them clean, the absence of fixed trash cans brings inconvenience to customers, resulting in poor hygiene and experience in the market.

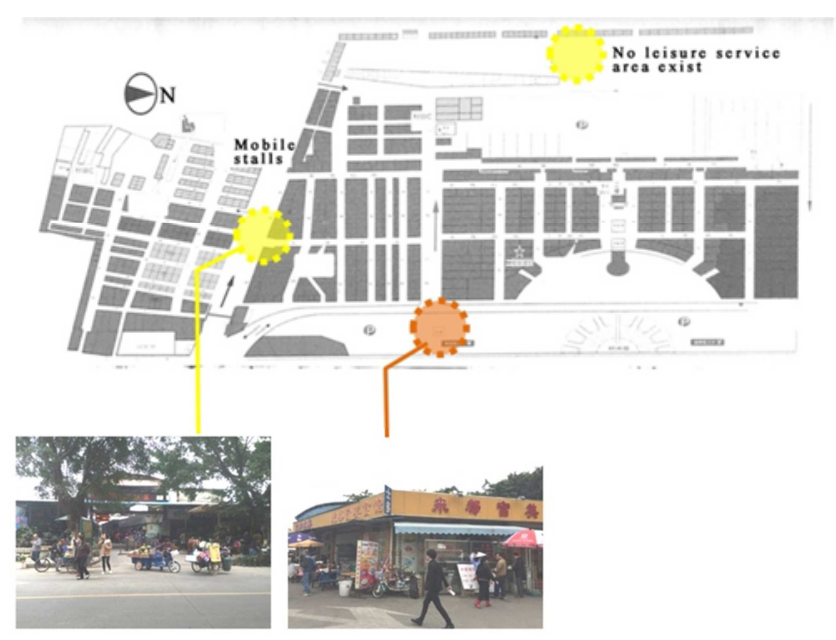

Figure 10. Leisure service area location.

\section{Specific Countermeasures}

Investigations suggest that two main problems exist in the architecture of Fangcun Flower and Bird Wholesale Market, lack of convenience and experience. And two reasons are concluded: the first is the shortage of architecture design planning of the wholesale market. Without a clear positioning at the very beginning of programming of the wholesale market, the expansion buildings are relatively independent in design and are not perfect in some details for specific functions. The second is the transition of the wholesale market from the traditional market model to the modern one. There is a lot to be improved for the wholesale market as changes always bring about new problems. Since built in 2001, the positioning of Fangcun Flower and Bird Wholesale Market given by the government of Guangzhou City has kept changing - from an ordinary flower and bird wholesale market to an important scenic spot and to the core of the large business circle. Each change of position requires more for its building design as the completed part can't satisfy the demand of its current position while its expansions are limited for no reserved land. Besides, as the expansion process of Fangcun Flower and Bird Wholesale Market lasted for a long time, many factors have changed, such as the design concepts, the design requirements, the design 
structures and the building materials, which have resulted in its current deficiencies in architecture design.

\subsection{Exterior Space Design of the Building}

The flower and bird wholesale market, as an important place for public entertainment, should be a complex of trade, exhibition, publicity, logistics and other functions rather than a single place for spot trading [1]. Therefore, its exterior space design should meet the various requirements of the users.

\subsubsection{Interface Design}

The design of entrance space of the flower and bird wholesale market should be put as the first consideration in this aspect. The entrance should be close to subway stations and bus stations, facilitating an easy flow of people; the number of entrance should be decided with reference to the function division and the built-up area of the market to meet the demands for gathering and evacuating of the crowds; the shape of the entrance, vital to a commercial building, is required to be eye-catching, strongly identifiable and harmonious with the whole building shape. Second is the design of disheartened space. Because the goods of the flower and bird wholesale market are mainly animals and plants, its disheartened space should be designed with more attention paid to the customer's experience and participation, increasing the purchase rate via more interaction between customers and goods; at the same time, the natural scenery should also be considered to provide a colorful and changeable shared space. The third consideration comes to the roof space. The roof, as the fifth elevation of a building, should be taken full advantage of to do vertical planting which helps to improve the flower and bird wholesale market's environment and mediate microclimate. The last is the billboard for investment, which should match with the building elevation and present flower and bird market characteristics, creating a new market image.

\subsubsection{Modeling Design}

Architectural modeling is particularly important in commercial buildings. The buildings of flower and bird wholesale market are usually single-story or multi-story, presenting a flat body. Thus, the modeling design is supposed to embody a combination of virtuality and reality by making a changing outer contour line. Then the design of its proportion and scale should be based on human activities to create comfortable shopping watching space and provide a sense of intimacy and security with its color and material.

\subsubsection{Environmental Design}

Differing from other ordinary markets, flower and bird wholesale market sells living creatures - flowers, plants, animals and birds, hence, its cleanness and environmental comfort, such as in sounds and scents, deserve to be emphasized. Moreover, it will be more attractive if combining potted flowers and plants goods with the market environment to create a safe and comfortable display space.

\subsection{Interior Space Design of the Building}

The functional components of the professional wholesale market cover six parts - operating facilities, ancillary facilities, transport facilities, service facilities, logistics facilities and public facilities [15]. And the wholesale market would be lack of sustained momentum if the interior space is characterized by disordered traffic, poor identifiability and short of a unified planning and supporting service facilities.

\subsubsection{Functional Configuration and Combination Mode}

First of all, the architecture of flower and bird wholesale market is unique in commercial buildings for its ornamental function, hence, apart from the basic function space of ordinary commercial buildings, the design should attach great importance to the exhibition, publicity and logistics function space. Secondly, the supporting service facilities of the buildings should meet the needs of all kinds of people, among which the upgrade and reconstruction of its pipeline layout, parking lots, groceries, food and beverage service area, rest area, and toilets are top priorities. The location of parking lots and entrances should be fully combined with the surrounding roads, and parking lot entrances should be marked clearly to give full play to their role as the instruction, which should also be expanded partly in case of congestion. All kinds of pipelines should be planned according to different zones, especially in the aquarium area, where the pipelines should be hidden inside or under the wall to ensure dry ground. Groceries and specifications for commodity should be standardized to meet the standard of groceries and the number should meet the needs of local businessmen and customers. Catering service area, as an indispensable part of commercial buildings, should be subject to a unified bidding by the flower and bird wholesale market, so the appropriate class of catering enterprises should be introduced to meet the daily catering needs. The rest area should be set up considering the adaptive design of the elderly and children and the integration of the public space and landscape. Toilets' layout position, area, quantity, daylighting, ventilation, squat number all directly affect the person's feeling while in use, which should be planned uniformly and cleaned regularly to keep it clean and tidy. The last one is to separate pedestrians from the vehicles and arrange the traffic streamline scientifically. The vehicles line can adopt three-dimensional transportation and stratified shunt, and the pedestrian line should be simple and convenient, with recognizability and accessibility. Simultaneously the monitoring system should be upgraded to improve the security of the interior of the buildings.

\subsubsection{Interior Space Composition}

The node space in commercial buildings is more complex, and it takes on many functions such as communication, traffic and stay, which should be enlarged locally and combined with natural environment to soften the conflicts. Street space is for hosting customers activities, which should be based on human activities and be built in safe and comfortable length, width, height, activating the sales 
activities of the whole street.

\subsubsection{Business Unit Space}

The demand for commercial unit space is different because of different regional commodities. When doing the building layout, investigation and survey aiming at stores' demands for all kinds of goods should be carried out, and the suitable column layout should be acquired after analysis. Layout made according to the modulus can increase the flexibility of the unit. Meanwhile, the accessibility of stores should be noticed to form a loop, avoiding blind angle.

\subsubsection{Fire Protection of Internal Space}

Commercial buildings are a gathering place for people, and fire safety is of great importance. Flower and bird wholesale market should be in strict accordance with the regulations to carry out fire partition, be designed with reasonable safety evacuation route, be equipped with fire doors, fire stairs, fire equipment, etc. according to the design specification partition to ensure the safety of people's life and property.

\subsection{Innovation of Wholesale Market Humanization, Informatization, Legalization and Branding}

The standardized modern wholesale market should have the functions of material collection and distribution, price generation, information release, standardization construction, service guidance, product promotion and mobilization [16]. At the beginning of the architectural design, research on flower and bird wholesale market should be conducted, and scientific and reasonable construction plan should be made combining with other subjects, aiming at building a new-type market of people-oriented with modern science and information technology, and providing a broad stage for the completed wholesale markets. At the same time, the management of the construction of wholesale markets should be accelerated for standardization and normalization, and the existing laws and regulations of the state, as well as regulations and industry regulations formulated by local departments and departments, should be improved as soon as possible. In addition, the wholesale market should study its advantages and expand marketing ideas and participate in and organize industry events and public welfare activities actively to promote its social image [17] and form a brand of one's own.

\section{References}

[1] Xie Dixiang, "Spacial distribution of wholesale market in metropolitan area as Guangzhou," Tropical Geography. China, vol.1, 2008, pp. 47-51.

[2] Cui Hong, and Li Yizhe, "The relocation of the Guanyuan flower and bird market," China Market. China, vol. z4, 2010, pp. 48-49.
[3] Zhu Xinsheng, "Yong'an flower and bird market, another eco-city highlights," Jiangyin Daily. China, vol. A01, July 12, 2006.

[4] Han Jian, and Jiang Yang, "The prosperous jewelry-flower-bird wholesale market in Kunming Old Streets," China Mining News. China, vol. B02, May 27, 2006.

[5] Xing Xiaowei, "The construction of large flower and bird market and the regulation of professional management," Zhoushan Daily. China, vol. 3, April 12, 2017.

[6] Liu Hongqi, and Lu Lin, "The thought-provoking relocation of flower and bird market: how to save folk culture amid urban development," Xinhua Daily. China, vol. 11, August 14, 2014.

[7] Xie Puqi, and Zhao Yang, "The transformation and development of the Qilou business street — as an example of the wholesale market in Guangzhou Yide road," Architecture \& Culture. China, vol. 10, 2017, pp. 226-228.

[8] Ma Hongzhao, "The analysis of the architectural design for the large-scale commodity wholesale market," Public Communication of Science \& Technology. China, vol. 3, 2014, pp. 48-49.

[9] Meng Zinan, and Zhang Yupeng, "The development status and countermeasures of commercial wholesale market- a case study of wenzhou mall in Gongzhuling city, the north of China," Sichuan Building Materials. China, vol. 2, 2017, pp. 71-72.

[10] Cui Yijun, and Lu Feiyu, "A brief discussion on fire characteristics and fire prevention of commodity wholesale market," Fire Community. China, vol. 8, 2016, p. 67.

[11] Lin Yu, "Optimization of safety evacuation design in large wholesale market," Inner Mongolia Science Technology \& Economy. China, vol.13, 2017, pp. 21-22.

[12] Zhao Yifan, and Li Xiaomin, "How to strengthen fire safety work in small commodity market," Shandong Industrial Technology. China, vol. 19, 2015, p. 273.

[13] Fang Ying, and Liu Chaoqiu, "An investigation of the flower folk in Fancun," Folklore Studies. China, vol. 1, 1998, pp. 47-50.

[14] Zhou Zhaoji, "A study of the floriculture in Guangzhou and Fangcun from a historical perspective," The Chinese Journal for the History of Science and Technology. China, vol.3, 1995, pp. 3-15.

[15] Wang Sufang, Xu Wei, and Zhang Jingjing, "Wholesale market planning and architectural space design: a case study on Zhejiang international mall," Technology Innovation and Application. China, vol. 4, 2015, p. 171.

[16] Liao Yanhua, and Cai Gennu, "Problems and countermeasures of agricultural products wholesale market in China," Xinjiang State Farms Economy. China, vol. 4, 2005, pp. 32-34.

[17] Shen Yujun, "Research and thoughts on development of Hefei Yufeng flower-bird-fish flea market," Legal System and Society. China, vol. 4, 2005, pp. 32-34. 